\title{
Hysteresis and Material Effects of Printed Strain Gauges Embedded in Organic Coatings ${ }^{\dagger}$
}

\author{
Herbert Enser 1,*, Johannes Kilian Sell 1, Michaela Schatzl-Linder ${ }^{2}$, Bernhard Strauß ${ }^{2}$, \\ Wolfgang Hilber ${ }^{1}$ and Bernhard Jakoby ${ }^{1}$ \\ 1 Institute for Microelectronics and Microsensors, Johannes Kepler University Linz, Linz, Austria; \\ Johannes_Kilian.Sell@jku.at (J.K.S.); Wolfgang.Hilber@jku.at (W.H.); Bernhard.Jakoby@jku.at (B.J.) \\ 2 R \& D voestalpine Stahl GmbH, Linz, Austria; Michaela.Schatzl@voestalpine.com (M.S.-L.); \\ Bernhard.Strauss@voestalpine.com (B.S.) \\ * Correspondence: herbert.enser@jku.at; Tel.: +43-732-2468-6262 \\ † Presented at the Eurosensors 2017 Conference, Paris, France, 3-6 September 2017.
}

Published: 17 August 2017

\begin{abstract}
This work reports on the hysteresis behavior and an initial baseline drift of printed strain gauges embedded in organic coating layers on sheet steel that we recently introduced. We subsequently investigated the performance over an extended period of time, which revealed interesting and partially unexpected material properties of printed strain gauges made from silver and carbon. Both silver- and carbon-based strain gauges show a hysteresis behavior of the gauge factor and non-negligible nonlinear characteristics. Furthermore, the carbon-based sensors show a strong initial base line drift within the first 50-100 cycles. All three effects, namely hysteresis, nonlinear gauge factor and initial base line drift, are confirmed within their respective standard deviations.
\end{abstract}

Keywords: printed sensors; embedded sensors; printed electronics; printed strain gauges; nonlinear temperature coefficients

\section{Introduction}

Strain gauges are state of the art for the detection and measurement of deformation (strain), stress or torque. In general, strain gauges are glued to the surface of a test device. This poses three main challenges: first, the adhesion layer may prevent proper transfer of the strain from the sample surface to the strain gauge sensor. This may, e.g., be the case if the adhesion layer is too soft or if the contact between strain gauge and adhesion layer is compromised, e.g., due to surface roughness, surface curvature, or impropriate application of the glue. Second, it may be difficult to find a suitable glue and apply the appropriate required pretreatment compatible with the surface. Typical pretreatment includes the cleaning of the surface with solvents and/or in addition sanding of the surface to enhance adhesion. Not all surfaces, especially polymeric ones, may be able to withstand these treatments. Third, applying a sensor to the surface is time consuming and wiring of the sensors is required. This can especially be difficult to handle and create significant costs, if a large number of sensors is to be applied to a surface.

To avoid these problems the application of strain gauges by printing techniques was investigated (e.g., [1-3]). Silver and carbon based printing pastes have proven to be appropriate for realization of such sensors. However, as recently reported [1] printed strain gauges exhibit a decrease of the gauge factor after the exposure to an additional temperature step. In addition to that further investigation of this behaviour revealed additional unexpected nonlinear responses to certain physical parameter changes such as temperature, as shown in [4]. Thus, this work will focus on the investigation of the nonlinear response of strain gauges when printed onto polymer coated rigid 
materials. Consequently, also the so-called "set-in" effect is discussed which occurs for different polymer based systems after different measurement cycles. To rule out effects coming from the organic polymer coating, a gold strain gauge is investigated as reference.

\section{Materials and Methods}

Strain Gauges investigated in this work are made of silver and carbon black polymer pastes and are fabricated via screen-printing. In addition to the previously mentioned two material systems, reference strain gauges are fabricated utilizing physical vapor deposition (PVD). This is done to investigate whether certain nonlinearities originate from the organic coating or if they can be attributed purely to the polymer inks. Figure 1 shows a schematic of a sample, where the substrate is sheet steel with an organic coating pre-applied for electric isolation $(1,2)$. On top of that, the sensor is realized (3). Optionally the sensor is then top-coated with another organic polymer layer utilizing spin-coating technology. This fabrication procedure was described in detail earlier $([1,5])$. The first and second strain gauge samples are printed with the following screen-print pastes: silver, (Henkel PF 050), and carbon black, (Henkel PR 406B). For the reference strain gauge sensor, PVD was utilized to deposit gold layers. While the first two material systems are based on particles embedded in polymer matrixes that require thermal curing, also the evaporated gold layer needs an additional annealing step to form a reliably stable strain gauge.

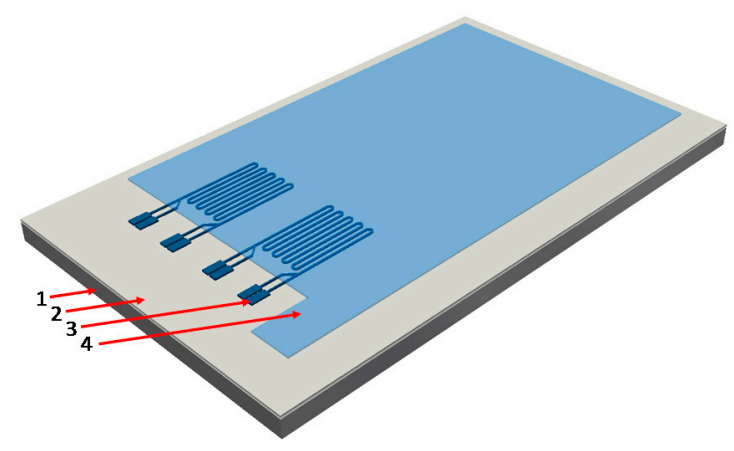

Figure 1. Schematic of printed strain gauges (3) on top of organic precoated (2) sheet steel (1), with an additional top coating layer (4) for mechanical protection and stabilization. The sensor layer (3) is fabricated either with screen-printing, for silver and carbon black, or deposited via PVD for gold.

\section{Discussion}

Characterization of the realized structures yielded that with silver and carbon black, sensor layer thicknesses of $\sim 4 \mu \mathrm{m}$ and $\sim 10 \mu \mathrm{m}$ were obtained, while the gold layer strain gauge has a thickness of $100 \mathrm{~nm}$. These geometric parameters results in an initial resistance of $340.7 \Omega$ for silver, 44,400 $\Omega$ for carbon black, and $150 \Omega$ for gold and a relative change in the range of \%o when compressive or tensile strain in the range of \%o is applied.

All measurements discussed in the following paragraph are conducted in controlled environmental conditions in a climate chamber WKL 100 from Weiss Technik. The parameters were set to $25^{\circ} \mathrm{C}$ for temperature and minimum humidity, which, according to the datasheet, corresponds to $25 \% \mathrm{RH}$ at $25{ }^{\circ} \mathrm{C}$. Figure 2 depicts one hundred measurement cycles of the silver-based strain gauge. One measurement cycle is defined as a movement of $+5 \mathrm{~mm}$ to impinge compressive strain onto the strain gauge, followed by a movement of $-10 \mathrm{~mm}$ to reach a $-5 \mathrm{~mm}$ position and thus impinge tensile strain resulting in 20 measurement points per cycle. It is clearly observable that after the first 10 cycles the silver-based strain gauge is more or less stabilized. On the contrary, as can be seen in Figure 3a showing the initial 100 cycles for the carbon black based strain gauge, it is evident that at least 90 are needed until the carbon black based sensor response can be presumed stable. To confirm this behaviour the same sample with carbon black was measured for a second time; again for 100 cycles. Figure $3 \mathrm{~b}$ depicts the second run for carbon black; it shows that the sensor response 
has stabilized significantly and no set-in effect can be observed. Further, the influence of sensor fabrication on the nonlinearity of the gauge factor was investigated. It was demonstrated that the nonlinearity could be significantly reduced by introducing a stabilizing top coat. This behavior is shown in Figure 4a,b. By introducing a top coat, the gauge factor of the silver strain gauge (compare Figure $4 \mathrm{~b}$ ) does not change significantly only the slight nonlinearities at the turning points vanish. The carbon-based sensor (Figure 4a) on the other hand becomes almost linear. In addition, its gauge factor is smaller after application of the top coat.

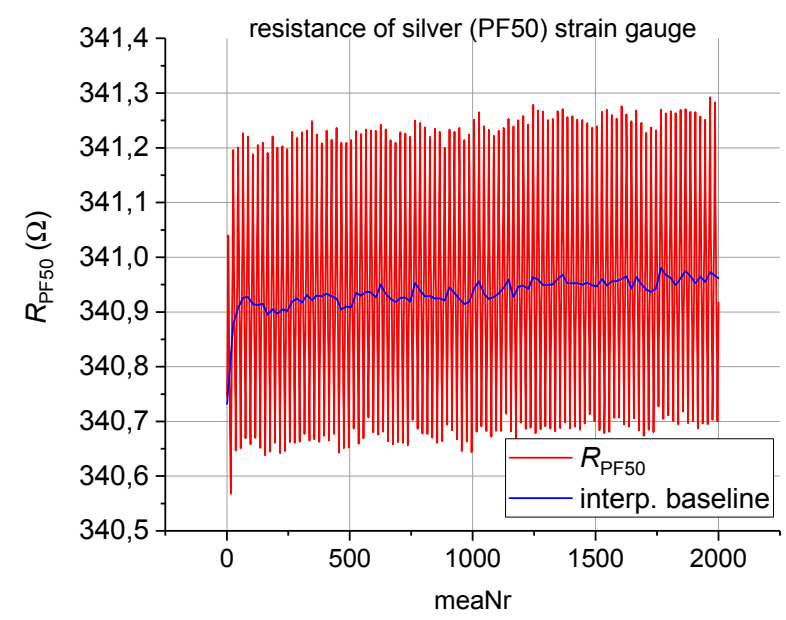

Figure 2. Silver ink based strain gauge measurement over 100 cycles, done at a constant temperature of $25^{\circ} \mathrm{C}$ and $25 \% \mathrm{RH}$ in a Weiss Technik WKL 100 climate chamber. No significant set-in effect is observed after the first 5-10 cycles, represented by the first 100-200 data points.

Finally, a hysteresis behavior that does not vanish after the initial baseline drift and remains after the application of a top coat is depicted in Figure $4 \mathrm{a}$ and Figure $4 \mathrm{~b}$. This suggests that some kind of rheological effect introduces this "lag" of the piezoresistive properties in case of the polymer-based inks. Furthermore Figure $4 \mathrm{a}, \mathrm{b}$ both depict an additional gauge factor measurement of pure gold. Since pure metallic strain gauges should respond linear and without hysteresis, the assumption that the hysteresis originates from the polymer coating can be made.

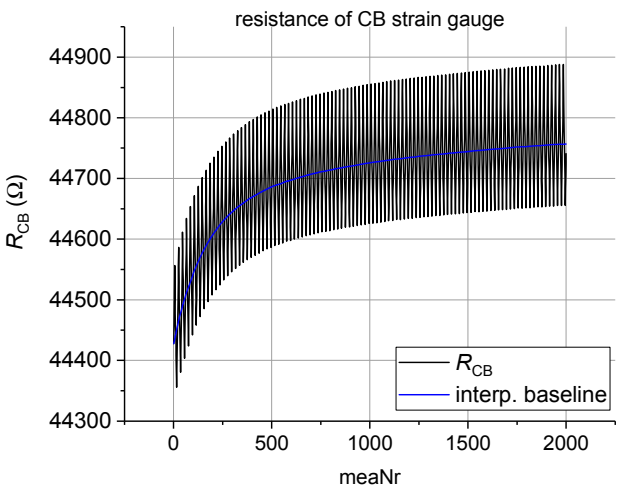

(a)

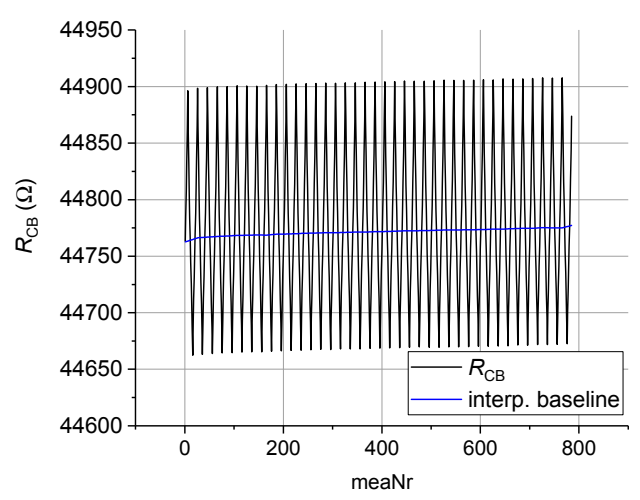

(b)

Figure 3. (a) Long-term measurement of 100 cycles for carbon black based strain gauge at a constant temperature of $25{ }^{\circ} \mathrm{C}$ and $25 \% \mathrm{RH}$ in a climate chamber. Only after about 90 out of 100 cycles, where one cycle amounts to 20 data points, the gauge factor becomes more stable; (b) Second long-term measurement for the same carbon black sample as in Figure 3a. The second run shows that the set-in period is completed. 


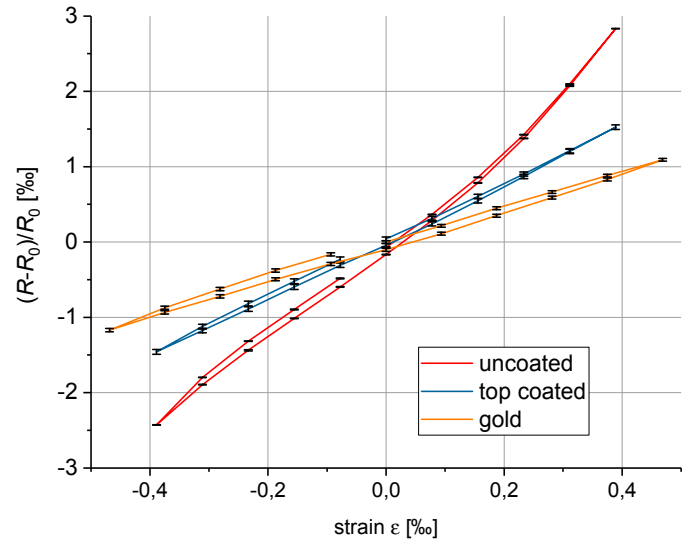

(a)

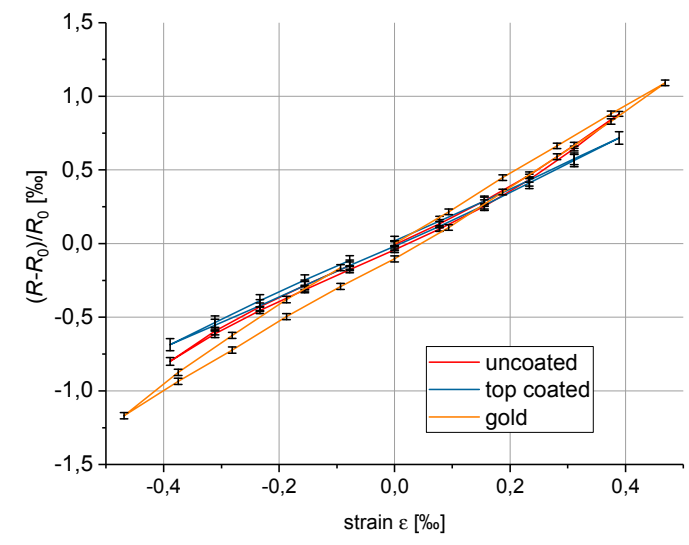

(b)

Figure 4. (a) Carbon-based strain gauges lose their nonlinearity after being top coated, but also the gauge factor decreases. Both measurements show a hysteresis effect, which is smaller than that for silver, though; (b) Silver-based strain gauges show a slight nonlinearity before top coating, which vanishes after application of the coating. Both measurements, however, show a hysteresis behavior for the gauge factor.

\section{Conclusions}

We showed that polymer based printed sensors need to be characterized thoroughly because they exhibit certain nonlinear effects. In addition, a mechanically stabilizing encapsulation is definitely improving the senor linearity, but with the slight drawback of reducing the sensor sensitivity. In conclusion, nonlinear effects can be attributed to the substrate coating since the comparison with a pure gold strain gauge showed the existence of a hysteresis as well.

Acknowledgments: This work has been supported by the Linz Center of Mechatronics (LCM) in the framework of the Austrian COMET-K2 programme and the Austrian. Research Promotion Agency (FFG-project No. 5834690).

Conflicts of Interest: The authors declare no conflict of interest.

\section{References}

1. Enser, H.; Kulha, P.; Sell, J.K.; Jakoby, B.; Hilber, W.; Strauß, B.; Schatzl-Linder, M. Printed Strain Gauges Embedded in Organic Coatings. Procedia Eng. 2016, 168, 822-825.

2. Rausch, J.; Salun, L.; Griesheimer, S.; Ibis, M. Printed piezoresistive strain sensors for monitoring of lightweight structures. In Proceedings of the SENSOR \& TEST Conference, Nurnberg, Germany, 6-9 June 2011; pp. 216-221.

3. Maiwald, M.; Werner, C.; Zoellmer, V.; Busse, M. INKtelligent printed strain gauges. Sens. Actuators A Phys. 2010, 162, 198-201.

4. $\quad$ Enser, H.; Kulha, P.; Sell, J.K.; Schatzl-Linder, M.; Strauß, B.; Hilber, W.; Jakoby, B. Printed strain gauges embedded in organic coatings-Analysis of gauge factor and temperature dependence. Unpublished work, 2017.

5. Sell, J.K.; Enser, H.; Jakoby, B.; Schatzl-Linder, M.; Strauss, B.; Hilber, W. Printed Embedded Transducers: Capacitive Touch Sensors Integrated into the Organic Coating of Metalic Substrates. IEEE Sens. J. 2016, 16, 7101-7108.

(C) 2017 by the authors. Licensee MDPI, Basel, Switzerland. This article is an open access article distributed under the terms and conditions of the Creative Commons Attribution (CC BY) license (http://creativecommons.org/licenses/by/4.0/). 